\title{
Análise da Conversação no Bate-papo: em Rede ou Centrada no Professor?
}

\author{
Valleska C. M. da Silva ${ }^{1}$, Mariano Pimentel ${ }^{1}$, Ricardo Rodriguez ${ }^{1}$, Vânia Maria \\ Félix Dias ${ }^{1}$ \\ ${ }^{1}$ Programa de Pós-Graduação em Informática - Universidade Federal do Estado do Rio \\ de Janeiro (UNIRIO) - Rio de Janeiro - RJ - Brasil \\ \{valleska.dasilva, pimentel, ricardo.rodriguez, vania\}@uniriotec.br
}

\begin{abstract}
Resumo. O bate-papo tem grande potencial para promover a interatividade no contexto da educação online. Ele foi desenhado como um meio de conversação que estabelece a relação todos-todos, possibilitando a conversação entre todos os interlocutores do grupo. Entretanto, quando usado no contexto educacional há a ameaça do professor se tornar o centro das atenções, promovendo uma utilização do bate-papo que se assemelha mais ao modelo um-todos. Para analisar a relação entre os sujeitos de uma sessão de bate-papo, foi proposto na presente pesquisa um modelo de análise da centralidade da conversação em sessões de bate-papo educacional, visando identificar se a conversação ocorreu centrada no professor ou em rede. Isso nos faz questionar as práticas pedagógicas online que estão sendo realizadas por meio do bate-papo: o professor-mediador está promovendo a colaboração entre os alunos ou está reforçando a assimetria comunicacional entre professor-aluno? Diferentemente do que se espera, muitas sessões de batepapo são realizadas de modo centrado no professor, conforme discutido nesta pesquisa.
\end{abstract}

\begin{abstract}
Chat has great potential to promote interactivity in the context of online education. It was designed as a way of conversation that establishes the all-all relationship, enabling conversation among all the interlocutors of the group. However, when used in the educational context there is the threat of the teacher becoming the center of attention, promoting a chat use that most resembles the one-all model. In order to analyze the relationship between the subjects of a chat session, a model of analysis of the centrality of the conversation in educational chat sessions was proposed in the present research, in order to identify if the conversation occurred centered in the teacher or if was collaborative. This makes us question the online pedagogical practices that are being carried out through the chat: is the mediator-teacher promoting collaboration among the students or reinforcing the communicational asymmetry between teacher-student? Different than expected, many chat sessions are conducted teacher-centered, as discussed in this research.
\end{abstract}

\section{Introdução}

A educação a distância tem crescido muito no Brasil (e também no mundo): de praticamente zero cursos no ano 2000, em pouco mais de uma década esta modalidade passou a ser responsável por quase 1/4 dos graduandos (23,4\%) (INEP/MEC, 2014). Dentre os meios de interação, o bate-papo é um dos mais utilizados (junto com fórum e 
email), sendo adotado por mais de $60 \%$ das instituições brasileiras que ofertam cursos a distância com interação online (Censo EAD, 2012), o que evidencia a importância de pesquisas sobre o uso de bate-papo na educação.

Diversos autores reconhecem o bate-papo como um meio para a realização de práticas pedagógicas (SILVA, 2010; STAHL, 2009; PIMENTEL et al., 2003). O batepapo possibilita a constituição de um espaço para realizar atividades diferentes da aula tradicional. A conversação informal, típica do bate-papo, possibilita o aluno perceber melhor o outro e perceber-se melhor como parte do grupo, proporciona um espaço para emoções que diminuem a sensação de isolamento tipicamente sentida na modalidade a distância. O uso contínuo do bate-papo integrado com atividades educacionais é uma forma de manter a motivação dos alunos e o engajamento para garantir o sucesso e continuidade de um curso online.

A presente pesquisa foi concebida sob o paradigma Design Science Research (DSR) em que se busca conhecer o mundo a partir do projeto e uso de artefatos artificiais (SIMON, 1969; HEVNER, 2010). Conforme destacado por Simon, o artefato é um elemento central nas pesquisas concebidas no paradigma DSR. O artefato é projetado para resolver um problema (objetivo do artefato) a partir de conhecimentos sobre o mundo (natural e social). $\mathrm{O}$ uso do artefato possibilita avaliar se parecem válidas as conjecturas teóricas do designer que subjazem o desenvolvimento do artefato. Desta forma, o processo de criação de um artefato adequado e a investigação sobre o seu uso num dado contexto se caracterizam como um meio para produzir conhecimento científico. (SIMON, 1996; DRESCHER et al., 2013; BAX, 2014). Nesta pesquisa, busca-se analisar e caracterizar a relação que efetivamente se estabelece entre os participantes durante uma sessão de bate-papo educacional. Com base nas conjecturas teóricas discutidas na seção 2 foi desenvolvido um artefato e, pelo seu uso podemos investigar e verificar se tais conjecturas são válidas. Na Seção 3 desta pesquisa são definidos os conceitos necessários para a compreensão da modelagem em grafo da troca de mensagens entre os interlocutores numa sessão de bate-papo, e as métricas de Análise de Redes Sociais (ARS) que adotamos. Além disso, ainda na Seção 3, propomos um algoritmo para caracterizar se a conversação estabelecida foi colaborativa ou centrada no professor. Na Seção 4, é apresentado o artefato desenvolvido nesta pesquisa, o RAC. Na seção 5, o algoritmo apresentado é usado para analisar logs reais de bate-papo ocorridos em contexto educacional. A conclusão desta pesquisa é apresentada na Seção 6.

\section{Bate-papo na Educação}

O modelo pedagógico adotado na maioria das salas de aula (presenciais e online) é baseado na cultura da transmissão de massa (modelo Um-Todos), isto é, o professor se posiciona como o detentor do conhecimento que deve ser transmitido, muitas vezes de maneira passiva, aos alunos. Esse modelo comunicacional ainda predominante nas instituições de ensino no Brasil, há décadas vem sendo criticado pelos pesquisadores em Educação. Paulo Freire, por exemplo, se referiu a esse modelo como a concepção "bancária" da educação, onde a única margem de ação dos alunos é receber depósitos de seus educadores e arquivá-los (FREIRE, 1974). Já Pierre (LÉVY, 1993) se referiu às escolas como uma instituição que "há cinco mil anos se baseia no falar/ditar do mestre".

A evolução da web 1.0 para web 2.0 na década de 20 vem ocasionando grandes mudanças na sociedade. Tais mudanças afetam não apenas as tecnologias digitais, mas o próprio cenário sociotécnico e comportamental de nossa sociedade. O ser humano do século XXI, deixa de ser reconhecido somente por sua aparência física e passa a ter sua 
identidade vinculada a um perfil, um endereço de correio eletrônico, um nickname, ele tem novos comportamentos, novos estilos de ser e agir, lê e escreve de forma diferente, desenvolveu novas formas de pensar, de aprender e de se relacionar com amigos. (NICOLACI-DA-COSTA; PIMENTEL, 2011, p.3). Na educação, observamos a mudança do paradigma da modalidade comunicacional massiva para a modalidade interativa. No cenário sociotécnico do século passado, o professor atuar como fonte de emissão de informação era compatível com o modelo de comunicação de massa característico daquele século, como exemplificado pelas TVs, rádios e jornais (modelo Um-para-Todos). Era, não é mais. Hoje em dia em tempos de cibercultura as pessoas incluindo os alunos demandam por interatividade num modelo de conversação todostodos. As pessoas já não ficam paradas, inerte em frente à tela da televisão; elas migram para a tela do computador, pois essa possibilita imersão, participação, modificação do conteúdo, e inevitavelmente educadores deverão repensar as instituições escolares e as práticas educativas que praticam. (NICOLACI-DA-COSTA; PIMENTEL, 2011).

Neste contexto, vários pesquisadores reconhecem o potencial do bate-papo como meio para a realização de práticas pedagógicas (SILVA, 2010; STAHL, 2009). O batepapo é um meio de conversação síncrona em que os usuários estão conectados simultaneamente e trocam mensagens textuais geralmente curtas. Ele estabelece a relação Todos-Todos entre os interlocutores (pois qualquer um pode enviar uma mensagem, e as mensagens enviadas são distribuídas para todos) conforme ilustrado na Figura 1.

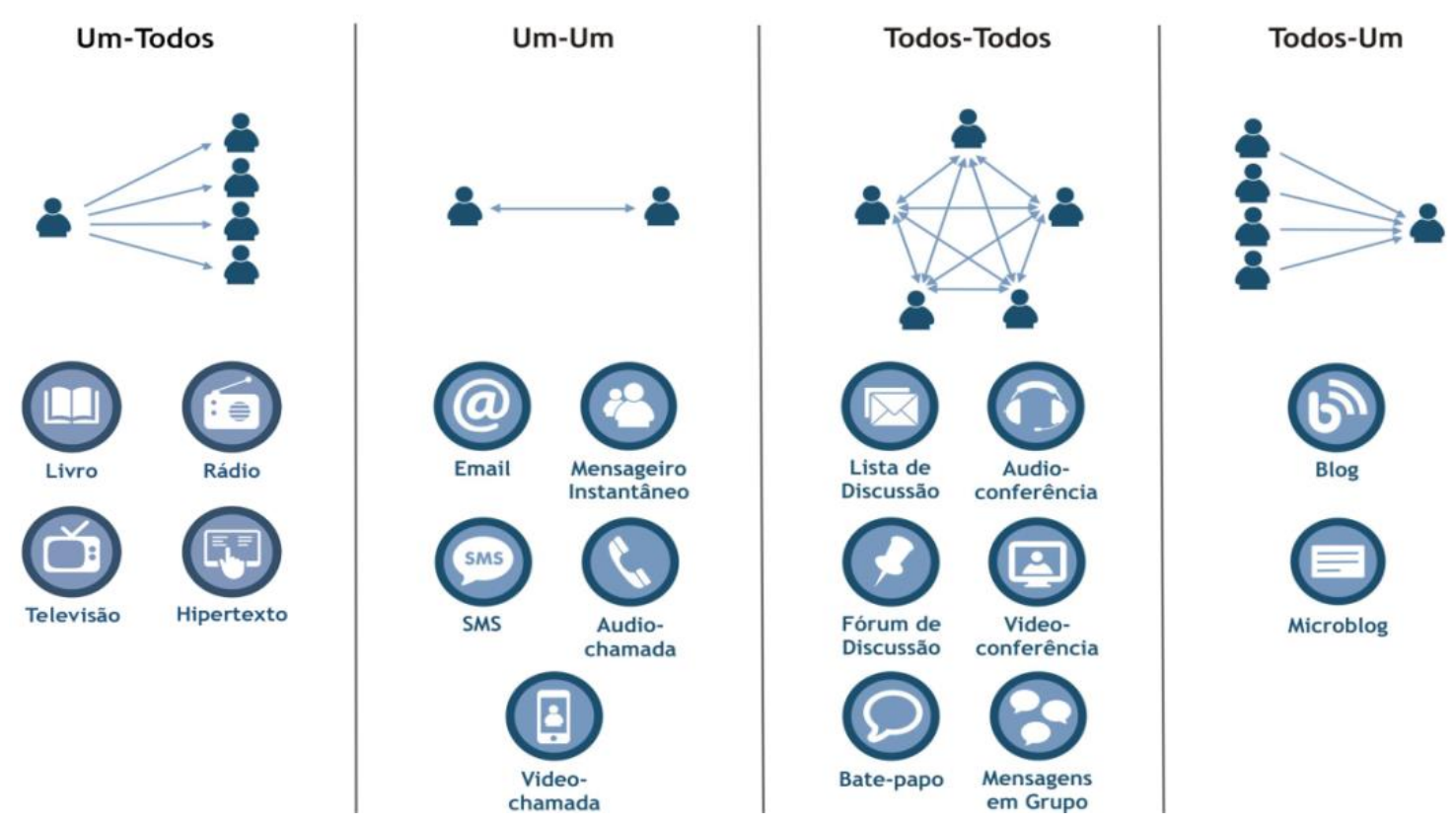

Figura 1. Relação entre os interlocutores nos diferentes meios de comunicação (baseado em Calvão et al., 2014)

Atualmente o bate-papo, junto com o email e o fórum de discussão, são os principais recursos online utilizados nos cursos a distância das instituições brasileiras (CENSOEAD.br, 2013). A inclusão do bate-papo no projeto pedagógico de um curso a distancia é fortemente indicado nos "Referenciais de qualidade para a educação superior a distância" da MEC (2007). Esse meio de conversação tem potencial para promover a motivação, a participação, e o engajamento dos alunos num curso a distância, além de promover a interatividade. Entretanto, para que se valorize o uso do bate-papo no 
contexto educacional, é preciso reconhecer que a educação não se restringe apenas a conteúdos ensinados numa abordagem intelectual e pragmática, mas que também envolve aspectos socioafetivos, as redes sociais e a colaboração, as emoções e o sentimento de pertença, dentre outros aspectos (MATURANA, 1998) que uma sessão de bate-papo tem potencial para promover na educação online, principalmente neste contexto em que os sujeitos do processo estão em espaços diferentes e ausentes de face.

Apesar do bate-papo ser um dos principais recursos online disponíveis nos cursos na modalidade a distância para promover a colaboração e a interatividade entre alunos (Todos-Todos), quando usado na educação pode ocorrer da conversação no batepapo ficar centrada no professor, reforçando a cultura da transmissão de massa (UmTodos) conforme ilustrado na Figura 2. Ainda que ocorra a conversa entre alunos, pode predominar a troca de mensagens dos alunos para o professor.

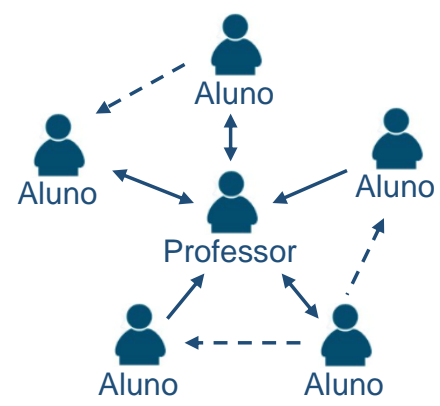

Figura 2. Conversação centrada no professor

Em um ambiente físico como uma sala de aula, é fácil perceber quando o professor é o centro das atenções. Entretanto, em ambientes virtuais como o bate-papo, essa percepção se torna mais difícil. Analisar a mediação online no bate-papo pode apoiar o professor a avaliar suas práticas pedagógicas. Foi com o objetivo de apoiar, computacionalmente, o professor a avaliar se mediou adequadamente a sessão de batepapo que essa pesquisa foi desenvolvida.

Para caracterizar se a conversação no bate-papo foi colaborativa ou centrada no professor, é preciso analisar as associações entre as mensagens para identificar quem falou com quem. Alguns sistemas que implementam o bate-papo possibilitam "responder" uma mensagem, como exemplifica o sistema DebatePapo (Moraes, 2011; Netto, 2014), e neste caso fica registrado a relação entre a mensagem do participante B para o participante $A(\mathbf{B} \rightarrow \mathbf{A})$. Alternativamente, a associação entre mensagens pode ser inferida pela análise do discurso. No presente artigo, foram usados $\log s$ com associações inferidas por um analista do discurso. A partir da associação de mensagens podemos gerar um grafo matemático e uma matriz de adjacência. Contabilizando a quantidade de mensagens trocadas entre os participantes da sessão, se for verificado uma predominância de mensagens destinadas ao professor, concluiremos que a conversação foi centrada no professor, caso contrário, concluiremos que a conversação foi colaborativa (em rede). Este método é formalizado na seção a seguir.

\section{Algoritmo para Identificação de Conversa Centrada no Professor}

As redes sociais são comumente representadas por meio de grafos. Um grafo, $\mathbf{G}=(\mathbf{V}, \mathbf{E})$, consiste em um conjunto finito e não vazio $\mathbf{V}$ de elementos chamados vértices e em um conjunto finito $\mathbf{E}$ de pares de elementos distintos de $\mathbf{V}$, chamados arestas. Cada um dos pares de $\mathbf{E}$ pode ser ordenado, no caso de grafo 
direcionado, ou não, no caso de grafo simples. Um multigrafo é um grafo que pode possuir arestas múltiplas, ou seja, uma ou mais arestas entre o mesmo par de vértices. Arestas múltiplas podem ser substituídas por uma aresta única com peso, sem prejuízo do seu significado. Neste caso, o peso de uma aresta corresponde ao total de arestas substituídas. O grau de um vértice é igual ao número de arestas incidentes sobre ele. Seja $\mathbf{G}$ um grafo direcionado, então se define grau de saída como o número de arestas partindo de um vértice e grau de entrada, o número de arestas chegando a um vértice. Um grafo pode ser representado por uma matriz de adjacência $\mathbf{A}$ onde $\mathbf{A}_{\mathbf{i}}$, j representa o número de arestas entre os vértices $\mathbf{i} \mathbf{e} \mathbf{j}$, para $\mathbf{i}=\mathbf{1}$..n e $\mathbf{j}=\mathbf{1}$..n, onde $\mathbf{n}$ denota o número de vértices de $\mathbf{G}$. No presente trabalho, $\mathbf{A}_{\mathbf{i}, \mathbf{j}}$ é igual ao número de arestas partindo de $\mathbf{i}$ para $\mathbf{j}(\mathbf{i} \rightarrow \mathbf{j})$. Denominaremos A por matriz de adjacência ponderada. Usando a matriz de adjacência ponderada $\mathbf{A}$ de $\mathbf{G}$, o grau de saída $\mathbf{S}_{\mathbf{i}}$ de um vértice $\mathbf{i}$ pode ser obtido somando a linha $\mathbf{i}$ de $\mathbf{A}$, ao passo que o grau de entrada $\mathbf{T}_{\mathbf{j}}$ de $\mathbf{j}$ é obtido somando a coluna $\mathbf{j}$ de $\mathbf{A}$.

Utilizaremos o grafo direcionado para representar a conversação numa sessão de bate-papo, como exemplificado na Figura 3, em que os vértices representam os participantes da sessão e cada aresta e seu respectivo peso representam quantas mensagens A enviou para B. O grau de saída representa quantas mensagens o participante $\mathbf{i}$ enviou. $\mathrm{O}$ grau de entrada representa quantas mensagens foram direcionadas para o participante $\mathbf{j}$.

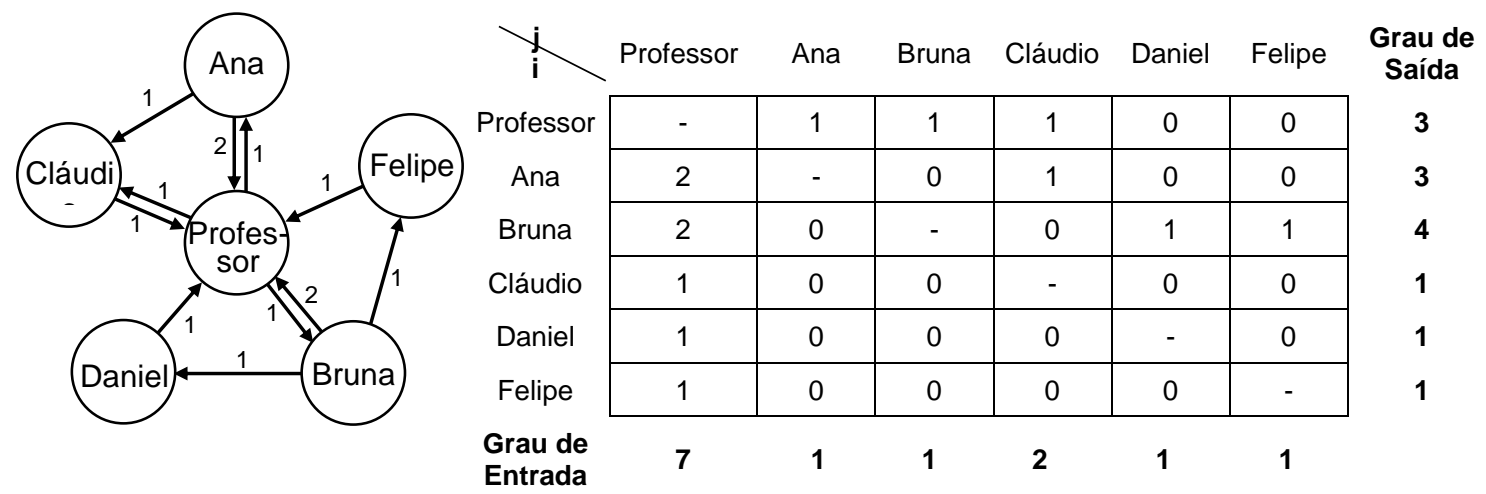

Figura 3. Grafo e Matriz de Adjacência representando um cenário de troca de mensagens num bate-papo

A análise de redes sociais é o campo que estuda as relações sociais entre atores e os padrões e implicações dessas relações. Uma rede social pode ser abstraída como um grafo, onde os atores são representados por vértices e as relações entre os mesmos são expressas por arestas, tal como exemplificado na Figura 3 com a modelagem da sessão de bate-papo por meio de um grafo direcionado sobre a troca de mensagens entre os participantes. É por meio da associação de cada mensagem (quem falou para quem) que mapeamos a rede social que se estabeleceu durante uma sessão de bate-papo. Estamos analisando, portanto, a rede social emergente de uma conversação (Recuero, 2012, p.143), especificamente da conversação realizada numa sessão de bate-papo.

Existem muitas métricas de centralidade para vértices individuais (Freeman, 2004). No contexto de bate-papo educacional, o número de participantes da sessão é reduzido (Calvão et al., 2014; Rocha et al., 2015) e assim muitas das métricas de centralidade não apresentam resultados significativos. Por esta razão, a métrica escolhida aqui foi a centralidade de grau, que é definida como o valor do grau de um vértice. Para este trabalho nos interessa em especial o grau de entrada, que indica a quantidade de mensagens recebidas por um participante (vértice). De acordo com 
Recuero (2012), além da importância do vértice, essa métrica pode indicar a popularidade e reputação de um determinado vértice na rede. Podemos ilustrar com o caso da Figura 3. Nessa figura, se nos concentrarmos no grau de saída, a aluna Bruna se destacará como uma participante central, pois foi a pessoa que mais enviou mensagens durante a sessão. Mas, apesar de ter falado bastante, Bruna não foi o foco de atenção daquela conversa, pois ao analisarmos o grau de entrada verificamos que Bruna está entre os que menos receberam mensagens, ou seja, ela não teve a atenção dos demais participantes. O foco da conversa era o professor, dado que para ele foram endereçadas muito mais mensagens do que para os demais participantes. Assim, o foco da conversação, neste contexto, é identificado através do grau de entrada e não pelo grau de saída.

Para analisar se a relação entre os interlocutores durante a sessão de bate-papo educacional foi centrada no professor, será utilizado o método de busca por Outlier_traduzido como "ponto fora da curva"_-_através da realização do teste de diagrama de caixas. Um ponto fora da curva é um valor que apresenta um grande afastamento dos restantes, é um valor aberrante, anormal comparado com os demais valores do conjunto. No contexto do bate-papo educacional, um ponto fora da curva acima do limite superior pode indicar que um participante foi o foco da conversação obtendo muito mais atenção que os demais. O Teste de Diagrama de Caixas é capaz de identificar se, num conjunto de dados, existem ou não pontos fora da curva:

1. Calcula-se a mediana, o quartil inferior $(\mathrm{Q} 1)$ e o quartil superior $(\mathrm{Q} 3)$;

2. Calcula-se $\mathrm{IQR}=(\mathrm{Q} 3-\mathrm{Q} 1)$

3. Calcula-se $Z=1,5 \times \mathrm{IQR}$

4. São classificados como pontos fora da curva os valores inferiores a $(\mathrm{Q} 1-\mathrm{Z})$ e superiores a $(\mathrm{Q} 3+\mathrm{Z})$.

No método de análise que elaboramos nesta pesquisa, só nos interessam os pontos fora da curva superiores, visto que a centralidade da conversação é caracterizada pela discrepante quantidade de mensagens endereçadas a alguém. A fim de identificar se a conversação no bate-papo foi ou não centralizada, propomos o seguinte algoritmo:

Algoritmo AnalisarCentralidade

Entrada: Grafo $\boldsymbol{G}=(\boldsymbol{V}, \boldsymbol{E})$ extraído de um dado Log de bate-papo.

Saída: Um conjunto vazio caso não seja centrado.

Um conjunto não vazio caso seja centrado.

Obter a matriz de adjacência $\boldsymbol{A}_{i, j}$ de $\boldsymbol{G}$;

Para cada $v \in V$, calcular o grau de entrada de $T v$;

Ordenar o vetor $T$ de modo crescente por valor do grau de entrada;

Aplicar a função Diagrama de Caixas:

Se houver pontos fora da curva escrever "bate-papo centrado" $e$ indicar centrado".

em quem esteve centrado; Caso contrário, escrever "bate-papo não

Caso a conversação seja centrada, o algoritmo descrito retorna quais são os pontos fora da curva, isto é, em qual ou quais pessoas esteve focada a conversação na sessão de bate-papo. Nesse caso, se o professor for o ponto fora da curva então concluímos que ele manteve o papel de detentor da atenção. 
VI Congresso Brasileiro de Informática na Educação (CBIE 2017)

Anais do XXIII Workshop de Informática na Escola (WIE 2017)

Aplicando o algoritmo proposto no log de bate-papo exemplificado na Figura 3, obtemos o seguinte resultado:

1. A matriz de adjacência ponderada $A$ de $G$ está representada na Figura 3.

2. O grau de entrada está representado na Figura 3.

3. Vetor T ordenado: $\{1,1,1,1,2,7\}$

4. Teste de Diagrama de Caixas em $\mathrm{T}$

○ $\quad$ Mediana $=1 ; \mathbf{Q 1}=1 ; \mathbf{Q 3}=1,75 ; \mathbf{I Q R}=0,75 ; \mathbf{Z}=1,125 ; \mathbf{Q 3}+\mathbf{Z}=2,875$

\section{Conversação centrada}

Portanto, seriam classificados como ponto fora da curva os valores superiores a 2,875 . Nesse caso, o valor 7 é detectado como ponto fora da curva por estar acima do valor 2,875. Este é o valor do grau de entrada do professor. Assim, concluímos que a conversação nesta sessão de bate-papo ocorreu de forma centrada no professor.

\section{RAC: Relatório de Análise da Conversação em bate-papo}

Como discutido na seção 1, pesquisas em DSR se destacam pelo desenvolvimento do artefato que é projetado para resolver um problema a partir de conhecimentos do seu campo de atuação. Nesta pesquisa foi desenvolvido o Relatório de Análise da Conversação (RAC) ilustrado na Figura 4, cujo objetivo é apresentar um resumo com as informações relevantes obtidas da análise do $\log$ da sessão de bate-papo visando apoiar o professor a avaliar seu desempenho como mediador da sessão, principalmente o alertando caso a conversação tenha sido centrada em algum participante (pretendemos alertar o professor que se posiciona no centro das atenções de um bate-papo). Esse artefato operacionaliza as conjecturas teóricas sobre a centralidade da conversação expressas em nosso modelo apresentado na seção anterior, de tal forma que, pelo uso do artefato, é possível investigar se as conjecturas teóricas parecem válidas e se o problema foi resolvido (se o professor consegue avaliar seu trabalho de mediação na sessão de bate-papo) - sendo estas as avaliações previstas na abordagem de pesquisa em Design Science Research.

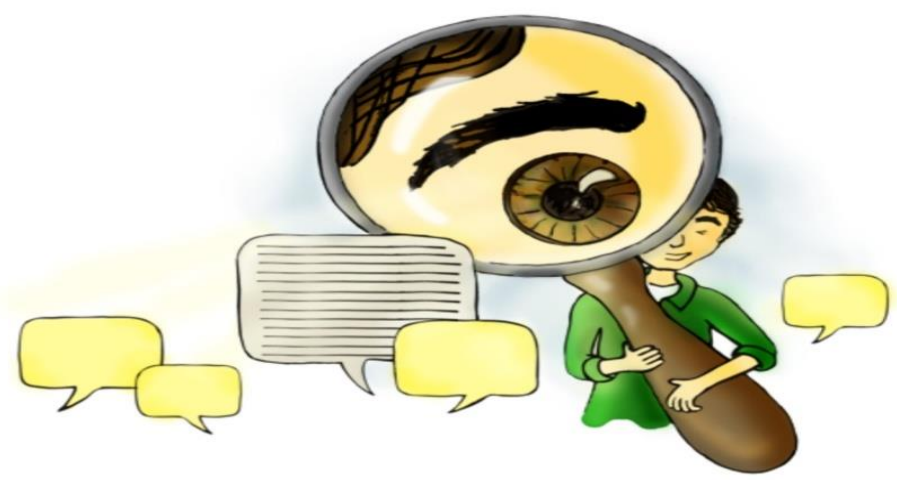

Análise da Conversação e da Rede Social
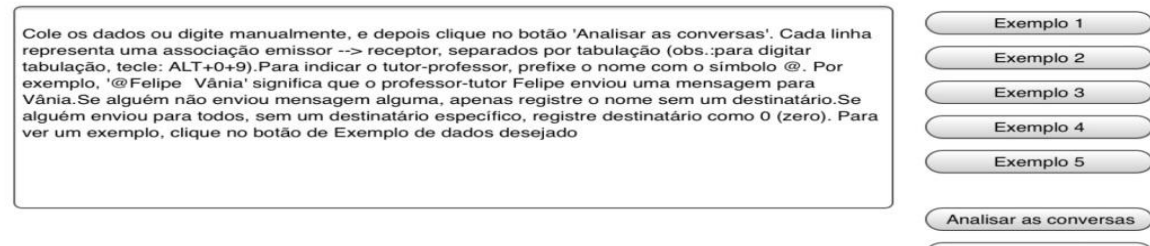

Limpar a tela

Figura 4. Página inicial do RAC 
O objetivo principal do RAC é informar a centralidade ou não da conversação. Entretanto, ele também apresentará outras informações relevantes. Ao clicar em "Analisar as conversas" a primeira e a segunda seção apresentam informações respectivamente da análise da produção de mensagens (mensagens enviadas pelos participantes) e da análise do endereçamento de mensagens (mensagens endereçadas aos participantes), figura 5 .

Análise de produção de mensagens

- Nesta sessão de bate-papo participaram 14 pessoas que trocaram 203 mensagens

- O grupo que enviou menos mensagens foi: Adilson(3 mensagens), Samara(3 mensagens), Jussara(0 mensagens) , Maria(6 mensagens) ,

- Atenção: Jussara, não enviou(aram) nenhuma mensagem.

- O grupo que mais enviou mensagens foi: Queli(30 mensagens), Viviane(41 mensagens), Tereza(29 mensagens), Roberta(21 mensagens),

- Atenção: Embora Viviane (41 mensagens) tenha enviado mais mensagens que os demais, esse valor não chega a ser discrepante do grupo!

Figura 5. Análise da produção de mensagens - RAC

Nas análises é informado o número de pessoas que participaram da sessão de bate-papo e o total de mensagens trocadas. São listadas as pessoas que menos enviaram/receberam mensagens (arbitrariamente cortadas até o primeiro quartil, que contém $25 \%$ dos valores mais baixos da amostra). É dado um alerta caso algum participante não tenha enviado/recebido mensagem. A última informação indica o resultado da análise de ponto fora da curva. No caso da análise da produção de mensagens um ponto fora da curva indica que alguém enviou muito mais mensagens que todos os outros, isto é foi o tagarela da turma. Já no caso do endereçamento de mensagens o ponto fora da curva indica se a conversa esteve centrada em alguém.

\section{Análise dos dados}

Aplicamos o algoritmo aqui desenvolvido em cinco $\log s$ reais de bate-papo educacional. Os $\log s$ são oriundos de uma disciplina do curso de Pedagogia em Licenciatura a distância da UNIRIO/CEDERJ/UAB. As sessões foram realizadas no bate-papo do Facebook. E as associações entre mensagens foram feitas através da análise do discurso. Os $\log s$ foram analisados através do artefato RAC, os resultados serão analisados na subseção a seguir.

\subsection{Logs Cederj}

A primeira sessão de bate-papo contava com dez participantes sendo um deles a tutora Gracy. Foi trocado um total de 71 mensagens. Três participantes não enviaram mensagens durante a sessão. A conversação ocorreu de modo centrado na tutora Gracy, pois um número discrepante de mensagens foi endereçado a ela, muito acima do que foi endereçado aos demais. O segundo log de bate-papo contava com quatorze participantes sendo um deles o tutor Ronaldo. Foi trocado um total de 209 mensagens. Um participante não enviou mensagens durante a sessão. E uma outra participante enviou muito mais mensagens que os demais alunos, de modo discrepante. A conversação ocorreu de modo centrado no tutor Ronaldo, pois um número discrepante de mensagens foi endereçado a ele, muito acima do que foi endereçado aos demais. O terceiro log de bate-papo contava com seis participantes sendo um deles a tutora Vivian. Foi trocado um total de 157 mensagens. Todos participaram da conversa! A conversação ocorreu de modo centrado no tutora Vivian, pois um numero discrepante de mensagens foi endereçado a ela, muito acima do que foi endereçado aos demais. O quarto log de batepapo contava com quatorze participantes sendo um deles a tutora Viviane. Foi trocado um total de 203 mensagens. Um participante não enviou mensagens durante a sessão. A 
conversação ocorreu de modo centrado na tutora Viviane, pois ela teve um número discrepante de mensagens endereçadas a ela. Por fim, o quinto log de bate-papo contava com quatorze participantes sendo um deles a tutora Simone. Foi trocado um total de 261 mensagens. Um participante não enviou mensagens durante a sessão. E uma outra participante enviou muito mais mensagens que os demais alunos, de modo discrepante. A conversação ocorreu de modo centrado na tutora Simone e em um outro aluno, pois um numero discrepante de mensagens foi endereçado a eles, muito acima do que foi endereçado aos demais.

\section{Conclusão}

A conversação em todos os $\log s$ analisados ocorreu de modo centrada no professor. O que nos leva a repensar o modelo educacional Um-para-Todos fortemente arraigado nos educadores do Brasil. Conforme observado com os resultados desta pesquisa, sem mediação adequada não é possível estabelecer a interatividade. Não adianta disponibilizar uma série de recursos e interfaces sofisticados se não houver uma mediação docente capaz de promover a interatividade. Para superar essa limitação dos docentes no Brasil, é preciso investir na inclusão digital e cibercultural do professor, isto é, o professor precisa ter acesso a um computador conectado à internet e aprender a lançar mão dos diferentes recursos online, conhecendo as funcionalidades de cada tecnologia e se apropriando delas para que possa explorá-las de acordo com as suas necessidades. Não se apropriar adequadamente das novas tecnologias significa andar na contramão da história, produzir exclusão social. De fato, o uso da internet na formação escolar e universitária é uma exigência da cibercultura.

Com esta pesquisa concluímos que o modelo proposto de análise da centralidade da conversação em bate-papo educacional, operacionalizado pelo artefato RAC mostrou-se adequado para caracterizar o modo como a conversação ocorre no batepapo. O modelo nos permitiu identificar se a conversação foi centrada no professor ou se foi estabelecida a colaboração entre os alunos. Como trabalho futuro, deverá ser feita a análise dos diferentes modelos de conversação que podem estar incluídos na conversação em rede. Também consideramos importante melhorar o artefato RAC e enriquecer o relatório com outras análises que possam complementar a compreensão dos professores em relação à conversação e sobre sua mediação numa sessão de batepapo.

\section{Referências}

Calvão, L. D., Pimentel, M., Fuks, H. Do Email ao Facebook: Uma perspectiva evolucionista sobre os meios de conversação da internet. Rio de Janeiro: UNIRIO, 2014.

CensoEAD. <http://www.abed.org.br/censoead/censoEAD.BR_2012_pt.pdf> Acesso em: 23 out 2014.

Estruc, M., Pimentel, M. (2012). Portal Tagarelas: bate-papo para educação. XXIII Simpósio Brasileiro de Informática na Educação - SBIE. Rio de Janeiro, RJ.

Freeman, L. C. (2004) The Development of Social Network Analysis. A Study in the Sociology of Science. Empirical Press: Vancouver, 2004.

Fuks, H. Aprendizagem e Trabalho cooperativo no Ambiente AulaNet. Revista Brasileira de Informática na Educação, n 6. abril de 2000. Disponível na Internet via Web: http://anauel.cead.puc-rio.br/aulanet/index.html 
VI Congresso Brasileiro de Informática na Educação (CBIE 2017)

Anais do XXIII Workshop de Informática na Escola (WIE 2017)

Inep (2012) Censo da Educação Superior 2012. MEC, 2012. Disponível em <http://portal.mec.gov.br/index.php?option=com_docman\&task=doc_download\&gid $=9332 \&$ Itemid $=>$. Acessadoem 20 nov 2014 .

Löber, A., Schwabe, G., Grimmi S (2007). Audio vs. chat: The effects of group size on media choice. Proceedings of the $40^{\text {th }}$ HICCS Hawaii International Conference on System Sciences.

Lucena, C. J. P., Fuks, H., et. al. Tecnologia de Informação Aplicada a Educação: Um Meta Curso no Ambiente AulaNet [online]. Monografia em Ciência da Computação, Departamento de Informática, PUC-Rio. Rio de Janeiro, março de 2000. Disponível em: 〈http://anauel.cead.pucrio.br/aulanet/index.html>

Moraes, E. L. C. (2011) "Debatepapo: sequências conversacionais e visualização do cotexto para compreensão da conversação em bate-papo”. 86 páginas. Dissertação de Mestrado. Departamento de Informática Aplicada, UNIRIO.

Netto, A.T.C. (2014) Sugestão de Associações entre Mensagens de Bate-papo: Um experimento com o sistema debatepapo V.2. Dissertação de Mestrado em Informática, UNIRIO, Rio de Janeiro, Brasil.

Pimentel, M.G., Fuks, H. e Lucena, C.J.P. (2003) "Debati, debati... aprendi? Investigações sobre o papel educacional das ferramentas de bate-papo", IX Workshop sobre Informática na Escola - WIE2003, Agosto.

Piva, D.J., Pupo, R., Gamez, L., Oliveira, S. (2011). EAD na Prática - Planejamento, métodos e ambientes de educação online. Elsevier Editora Ltda., 2011, v.,p 164-170.

Recuero, R. (2012) A conversação em rede: comunicação mediada pelo computador e redes sociais na Internet - Porto Alegre: Ed. Sulina.

Rocha, E.B., Pimentel, M., Diniz, M.C. Quantidade de Participantes em Bate-papo Educacional: um Modelo Baseado em Teoria de Filas. Revista Brasileira de Informática na Educação (no prelo). 2015.

Silva, M. (2010) Avaliação da Aprendizagem em Educação Online. 2010. Entrevista publicada no Youtube<http://youtu.be/S7uUd6afEYE>, acessado em 18 abr 2015.

Stahl, G. (Ed.). (2009). Studying virtual math teams. New York, NY: Springer. 